\title{
Pathologic von Willebrand factor degradation is a major contributor to left ventricular assist device-associated bleeding: pathophysiology and evolving clinical management
}

\author{
Carlo R. Bartoli \\ Division of Cardiovascular Surgery, Hospital of the University of Pennsylvania, Philadelphia, PA, USA \\ Correspondence to: Carlo R. Bartoli, MD, PhD. Division of Cardiovascular Surgery, Hospital of the University of Pennsylvania, 380 South University \\ Ave., Hill Pavilion, Room 410B, Philadelphia, PA 19104, USA. Email: carlo.bartoli@uphs.upenn.edu.
}

Submitted Nov 05, 2020. Accepted for publication Jan 18, 2021.

doi: 10.21037/acs-2020-cfmcs-29

View this article at: http://dx.doi.org/10.21037/acs-2020-cfmcs-29

\section{Introduction}

Non-surgical bleeding is the most frequent adverse event in patients with a continuous-flow left ventricular assist device (CF-LVAD). Bleeding is a major source of morbidity and an obstacle to improving outcomes, reducing costs, and expanding the public health impact of LVAD therapy. Mounting evidence demonstrates that the acquired von Willebrand factor (VWF) deficiency caused by CF-LVAD support is an important contributor to bleeding.

\section{Epidemiology}

Thirty to seventy-five percent of LVAD patients experience episodic bleeding (1-4). Twenty to forty percent of patients bleed from the alimentary tract, most commonly from angiodysplasia $(3,4)$. Gastrointestinal bleeding is the number one cause of hospital readmission for ambulatory LVAD patients. This is in part because all CF-LVAD patients acquire VWF deficiency (1-5). Within minutes of LVAD support, pathologic VWF degradation occurs. Within 24 hours, VWF deficiency plateaus and remains until LVAD support is discontinued.

\section{Normal VWF physiology}

VWF, a multimeric plasma clotting protein, initiates primary hemostasis. Shear stress closely regulates VWF activation for thrombogenesis (6). Normal intravascular shear stress is approximately 2 to $8 \mathrm{~Pa}$. VWF activation occurs above $10 \mathrm{~Pa}$. During activation, shear stress unravels inactive, globular VWF into an active, elongated conformation, which exposes binding sites for collagen and platelets to generate thrombus. Importantly, highmolecular-weight VWF multimers are most functional for clotting. However, unraveled, active high-molecular-weight VWF multimers are also sensitive to enzymatic cleavage by ADAMTS-13, the VWF-specific protease. ADAMTS-13 degrades active high-molecular-weight VWF multimers into low-molecular-weight VWF multimers and VWF fragments that are ineffective in clotting.

\section{LVAD-induced VWF degradation}

CF-LVADs generate shear stress that exceeds physiologic values by two orders of magnitude (6). Inside CF-LVADs, an impeller spins at thousands of revolutions per minute (RPM) to propel blood forward. Shear stress of up to $1,500 \mathrm{~Pa}$ is produced. As a result, supraphysiologic LVAD shear stress causes pathologic VWF degradation by two biophysical mechanisms (2): (I) major mechanism-mechanoenzymatic degradation: LVAD shear stress activates VWF and exposes ADAMTS-13 cleavage sites for enzymatic degradation. (II) Minor mechanism-non-enzymatic degradation: shear stress alone (independent of ADAMTS-13) tears VWF multimers into smaller multimers. In parallel, LVAD shear stress activates platelets, which partially degranulate and release stores of ultra-high-molecular-weight VWF multimers (7), an important VWF reserve. Consequently, in 
patients with an LVAD, platelet VWF is partially depleted when needed for primary hemostasis.

\section{Relationships between VWF deficiency and bleeding}

A causal relationship between absence of high-molecularweight VWF multimers and bleeding is well established. Non-LVAD patients with type IIA (congenital) and type IIB (acquired) von Willebrand Syndrome bleed because functional VWF multimers are absent. Patients often present with gastrointestinal bleeding from angiodysplasia $(3,4)$. Similarly, in patients with Heyde's syndrome, elevated shear stress through a stenotic aortic valve causes degradation of high-molecular-weight VWF multimers and bleeding from angiodysplasia. Mechanisms of VWF degradation in Heyde's syndrome and LVAD patients are nearly identical (1-4).

Likewise, laboratory derangements and clinical presentation of LVAD patients are similar to patients with type II von Willebrand's disease and Heyde's syndrome. Patients exhibit absence of high-molecular-weight VWF multimers, reduced VWF: collagen binding and Ristocetininduced platelet aggregation, and prolonged bleeding times that are independent of INR and antiplatelet therapy. Not surprisingly, a dose-response relationship exists. LVAD patients with the least high-molecular-weight VWF multimers and lowest VWF function are most likely to bleed (8).

\section{Pathologic VWF metabolism may contribute to angiodysplasia}

CF-LVAD patients develop nasal mucosal vascular proliferation and gastrointestinal arteriovenous malformations $(1,3,4)$. Emerging evidence suggests this is a distinct form of angiodysplasia $(3,4)$, which begs the question, does abnormal VWF metabolism somehow alter angiogenesis and cause angiodysplasia? Interestingly, VWF is an early regulator of angiogenesis. In fact, VWF interacts with at least twenty molecular partners involved in angiogenesis, vascular inflammation, endothelial life cycle, smooth muscle proliferation, and arterial remodeling $(1,3,9)$. Indeed, normal angiogenesis requires normal levels of VWF multimers. In contrast, absence of VWF multimers and high levels of VWF fragments may alter angiogenesis and cause angiodysplasia in multiple human diseases $(1,3,4,9)$. As a result, in CF-LVAD patients, pathologic VWF degradation is likely a strong contributor to the high incidence of gastrointestinal angiodysplasia and bleeding (3).

\section{Management}

Consensus guidelines do not exist to manage LVADassociated bleeding. Blood transfusion may be lifesaving, but sensitizes bridge-to-transplant candidates. Reduced anticoagulation and cessation of antiplatelet therapy prevent recurrent bleeding but increase risk of LVAD thrombosis. Endoscopic intervention for gastrointestinal bleeding addresses sources of bleeding but does not address underlying pathophysiology. Likewise, pharmacotherapy with Octreotide, Arginine Vasopressin, Danazol, synthetic Estrogens, and Wilfactin have demonstrated limited success in managing LVAD bleeding, likely because pathophysiologic mechanisms of VWF degradation and angiodysplasia are not specifically targeted (3).

Targeted therapies designed to preserve VWF multimers (10) or to correct angiogenic imbalance (3) may have clinical utility. As an example, ADAMTS-13 inhibition is a potential strategy to reduce pathologic VWF degradation during high shear stress. However, this strategy must be approached with caution. Accumulation of activated, pro-thrombotic VWF multimers has potential to cause thrombosis (11). Alternatively, thalidomide, a potent anti-angiogenesis agent, may prevent recurrent bleeding from angiodysplasia in LVAD patients.

Reduction of LVAD RPM has been proposed as an adjunctive therapy for LVAD-associated bleeding. However, clinical and experimental data demonstrate that RPM reduction does not reduce shear stress sufficiently to preserve VWF multimers and is inappropriate bleeding management (12). In contrast, pulsatile blood flow may protect against LVAD-associated bleeding (13). Pulsatile stretch is an important trigger for endothelium to secrete VWF multimers and replenish plasma VWF (14). Pulsatile flow through the aortic valve also maintains a normal flow path in parallel with the LVAD pathway, which may reduce total blood exposure to supraphysiologic shear stress through the LVAD (12).

Ultimately, LVADs designed with reduced shear stress and pulsatile flow may reduce bleeding (6). Specific LVAD design features (impeller shape/orientation, RPM range, 
flow-gap size, internal geometry, blood transient time, flow algorithm) influence shear stress. As such, next-generation LVADs designed to minimize shear stress and preserve VWF multimers should reduce bleeding.

\section{Toward standardized testing of VWF degradation during mechanical circulatory support}

Multiple qualitative, semi-quantitative, and quantitative techniques to evaluate the severity of VWF deficiency in LVAD patients have been reported (5). However, a standard methodology has not been established as has been for hemolysis. As a result, interpretation of different metrics of VWF multimer degradation across preclinical and clinical studies is limited. Therefore, a consensus practice to quantify and report VWF multimers and industry standards for LVAD-associated VWF degradation is needed to standardize comparisons across patients, devices, studies, and clinical outcomes (5).

\section{Conclusions}

CF-LVADs cause pathologic VWF degradation, acquired VWF deficiency, gastrointestinal angiodysplasia, and recurrent bleeding. Management strategies are evolving. Targeted therapies designed to preserve VWF multimers or to correct angiogenic imbalance may have clinical utility. Next-generation LVADs designed with lower shear stress and pulsatile flow will prevent abnormal VWF metabolism and reduce LVAD-associated bleeding. Standardized testing and accepted thresholds for VWF degradation in LVAD patients are needed.

\section{Acknowledgments}

Funding: None.

\section{Footnote}

Conflicts of Interest: The author has no conflicts of interest to declare.

Open Access Statement: This is an Open Access article distributed in accordance with the Creative Commons Attribution-NonCommercial-NoDerivs 4.0 International
License (CC BY-NC-ND 4.0), which permits the noncommercial replication and distribution of the article with the strict proviso that no changes or edits are made and the original work is properly cited (including links to both the formal publication through the relevant DOI and the license). See: https://creativecommons.org/licenses/by-nc-nd/4.0/.

\section{References}

1. Proudfoot AG, Davidson SJ, Strueber M. von Willebrand factor disruption and continuous-flow circulatory devices. J Heart Lung Transplant 2017;36:1155-63.

2. Bartoli CR, Restle DJ, Zhang DM, et al. Pathologic von Willebrand factor degradation with a left ventricular assist device occurs via two distinct mechanisms: mechanical demolition and enzymatic cleavage. J Thorac Cardiovasc Surg 2015;149:281-9.

3. Bartoli CR, Zhang DM, Hennessy-Strahs S, et al. Clinical and in vitro evidence that left ventricular assist device-induced von Willebrand factor degradation alters angiogenesis. Circ Heart Fail 2018;11:e04638.

4. Kang J, Hennessy-Strahs S, Kwiatkowski P, et al. Continuous-flow LVAD support causes a distinct form of intestinal angiodysplasia. Circ Res 2017;121:963-9.

5. Hennessy-Strahs S, Bermudez CA, Acker MA, et al. Toward a standard practice to quantify von Willebrand factor degradation during LVAD support. Ann Thorac Surg 2020. [Epub ahead of print]. doi: 10.1016/ j.athoracsur.2020.09.039.

6. Bartoli CR, Hennessy-Strahs S, Gohean J, et al. A novel toroidal-flow left ventricular assist device minimizes blood trauma: implications of improved ventricular assist device hemocompatibility. Ann Thorac Surg 2019;107:1761-7.

7. Dassanayaka S, Slaughter MS, Bartoli CR. Mechanistic pathway(s) of acquired von willebrand syndrome with a continuous-flow ventricular assist device: in vitro findings. ASAIO J 2013;59:123-9.

8. Hennessy-Strahs S, Bermudez CA, Acker MA, et al. Toward a standard practice to quantify von Willebrand factor degradation during LVAD support. Ann Thorac Surg 2020. [Epub ahead of print]. doi: 10.1016/ j.athoracsur.2020.09.039.

9. Randi AM, Smith KE, Castaman G. von Willebrand factor regulation of blood vessel formation. Blood 2018;132:132-40. 
10. Bartoli CR, Kang J, Restle DJ, et al. Inhibition of ADAMTS-13 by doxycycline reduces von Willebrand factor degradation during supraphysiological shear stress: therapeutic implications for left ventricular assist deviceassociated bleeding. JACC Heart Fail 2015;3:860-9.

11. Bartoli CR, Zhang D, Kang J, et al. Clinical and in vitro evidence that subclinical hemolysis contributes to LVAD thrombosis. Ann Thorac Surg 2018;105:807-14.

12. Kang J, Zhang DM, Restle DJ, et al. Reduced continuousflow left ventricular assist device speed does not decrease

Cite this article as: Bartoli CR. Pathologic von Willebrand factor degradation is a major contributor to left ventricular assist device associated bleeding: pathophysiology and evolving clinical management. Ann Cardiothorac Surg 2021;10(3):389-392. doi: 10.21037/acs-2020-cfmcs-29 von Willebrand factor degradation. J Thorac Cardiovasc Surg 2016;151:1747-54.e1.

13. Wever-Pinzon O, Selzman CH, Drakos SG, et al. Pulsatility and the risk of nonsurgical bleeding in patients supported with the continuous-flow left ventricular assist device HeartMate II. Circ Heart Fail 2013;6:517-26.

14. Vincent F, Rauch A, Loobuyck V, et al. Arterial pulsatility and circulating von Willebrand factor in patients on mechanical circulatory support. J Am Coll Cardiol 2018;71:2106-18. 